\title{
Syndrome paranéoplasique vasculaire touchant les extrémités
}

\author{
Philipp Jud MD, Reinhard B. Raggam MD, Franz Hafner MD
}

- Citation : CMAJ 2020 November 16;192:E1470. doi : 10.1503/cmaj.200430-f

Voir la version anglaise de l'article ici : www.cmaj.ca/lookup/doi/10.1503/cmaj.200430

$\mathbf{U}$

n homme de 85 ans ayant des antécédents d'adénocarcinome pulmonaire non à petites cellules récidivant a consulté à la clinique pour une cyanose et une nécrose douloureuses aux doigts depuis 2 semaines (figure 1 ), accompagnées d'une perte de poids, d'une toux sèche et de dyspnée. Il avait reçu un diagnostic de maladie de Raynaud (non strictement liée à l'exposition au froid) 5 ans plus tôt, et présenté des ulcères digitaux à répétition au cours des 2 dernières années. Il avait également subi une lobectomie pour son cancer du poumon 10 ans auparavant; une récidive a été diagnostiquée 2 mois avant sa consultation.

À l'examen physique, le patient présentait une cyanose et une nécrose des extrémités, et ses pouls ulnaires bilatéraux étaient non palpables. Il ne présentait aucun autre signe de maladie des tissus conjonctifs ou de vascularite. Les résultats des analyses de laboratoire étaient normaux (formule sanguine complète, vitesse de sédimentation, coagulation, analyse d'urine, et recherche d'anticorps antihépatite $B$ et $C$, de cryoglobulines et de plusieurs autoanticorps), à l'exception d'une augmentation du taux de protéine C-réactive $(99,1 \mathrm{mg} / \mathrm{L}$ [valeurs de référence : 0-5 $\mathrm{mg} / \mathrm{L}])$. L'échographie duplex couleur a révélé une occlusion bilatérale des artères ulnaires et palmaires; la capillaroscopie périunguéale était sans particularités. Nous avons diagnostiqué un syndrome paranéoplasique vasculaire touchant les extrémités et traité le patient de manière palliative au moyen d'iloprost $20 \mu \mathrm{g}$ intraveineux pendant 10 jours et d'hydromorphone orale $1,3 \mathrm{mg}$ au besoin. La nécrose s'est étendue aux pieds 2 semaines après la consultation, et le patient est décédé 1 mois plus tard (annexe 1, accessible en anglais au www.cmaj.ca/lookup/ doi/10.1503/cmaj.200430/tab-related-content).

Le syndrome paranéoplasique vasculaire touchant les extrémités est une maladie vasculaire rare. Dans $50 \%$ des cas, il est associé à un adénocarcinome, sans prédominance liée au sexe $\mathrm{e}^{1,2}$. Il s'accompagne d'ischémie digitale, ce qui comprend la maladie de Raynaud, d'acrocyanose et de gangrène digitale $e^{1,3}$. Selon une revue rétrospective de 15 cas, l'intervalle médian entre le diagnostic de cancer et

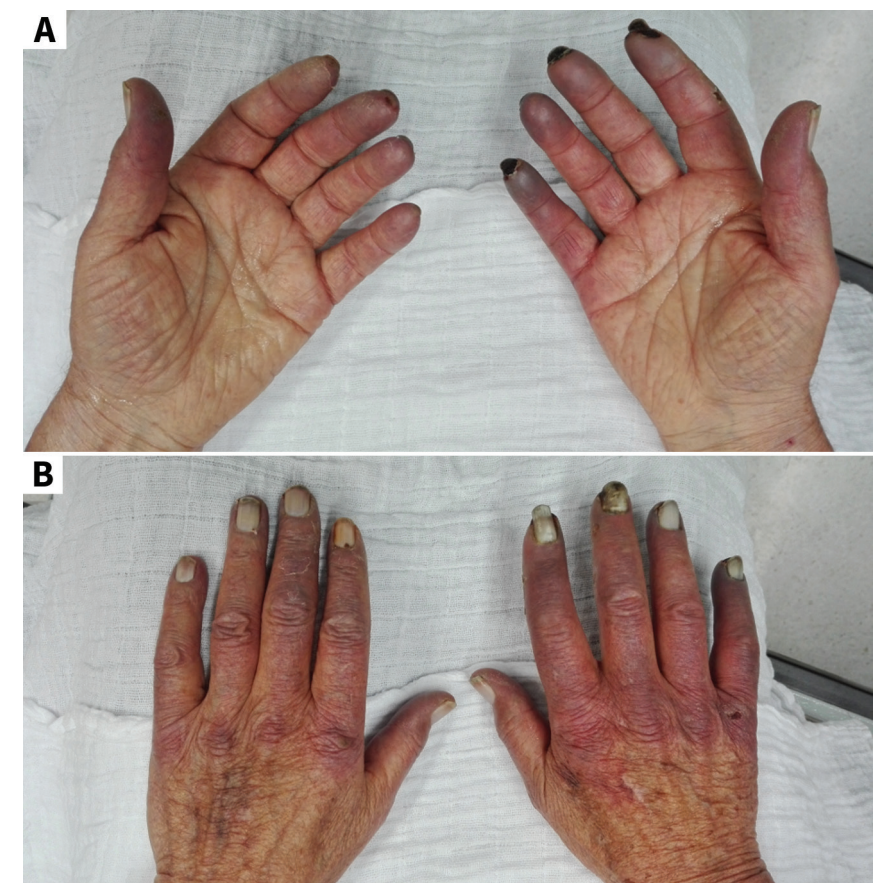

Figure $1:$ A) Cyanose de l'extrémité des doigts d'un homme de 85 ans, avec nécrose de l'index, du majeur, de l'annulaire et de l'auriculaire droits, et du pouce, de l'index, du majeur et de l'annulaire gauches. B) Coloration livide de la peau de la face dorsale des doigts, de l'index à l'auriculaire, et des articulations métacarpophalangiennes.

la nécrose des extrémités est de 2 mois (intervalle: 1-9 mois $)^{2}$. La gangrène digitale semble commencer au même moment que le cancer ou plus tard, mais elle peut aussi le précéder. Plusieurs mécanismes ont été avancés, notamment l'envahissement tumoral du système nerveux sympathique, la microembolisation de fragments tumoraux, ainsi que l'hyperviscosité sanguine et la surproduction de médiateurs vasoconstrictifs, thrombogènes et immunologiques par les cellules tumorales ${ }^{1,4,5}$. La plupart des cas rentrent dans l'ordre lorsque la tumeur primitive est traitée. Les prostaglandines peuvent offrir un soulagement ${ }^{2}$. 


\section{Références}

1. Poszepczynska-Guigné E, Viguier M, Chosidow O, et al. Paraneoplastic acral vascular syndrome: epidemiologic features, clinical manifestations, and disease sequelae. J Am Acad Dermatol 2002;47:47-52.

2. Le Besnerais M, Miranda S, Cailleux N, et al. Digital ischemia associated with cancer: results from a cohort study. Medicine (Baltimore) 2014;93:e47.

3. Chow SF, McKenna $\mathrm{CH}$. Ovarian cancer and gangrene of the digits: case report and review of the literature. Mayo Clin Proc 1996;71:253-8.

4. Hsu ST, Lee YY, Lie MF. Symmetrical peripheral gangrene of sudden onset a paraneoplastic syndrome? - a case report and review of the literature. Dematol Sinica 1996;14:82-8.

5. Falanga A, Marchetti M, Vignoli A. Coagulation and cancer: biological and clinical aspects. J Thromb Haemost 2013;11:223-33.

\section{Intérêts concurrents : Aucun déclaré.}

Cet article a été révisé par des pairs.

Les auteurs ont obtenu le consentement du patient.

Affiliation : Division d'angiologie, Département de médecine interne, Université médicale de Graz, Graz (Autriche).

Propriété intellectuelle du contenu : Il s'agit d'un article en libre accès distribué conformément aux modalités de la licence Creative Commons Attribution (CC BY-NC-ND 4.0), qui permet l'utilisation, la diffusion et la reproduction dans tout médium à la condition que la publication originale soit adéquatement citée, que l'utilisation se fasse à des fins non commerciales (c.-à-d., recherche ou éducation) et qu'aucune modification ni adaptation n'y soit apportée. Voir : https://creativecommons.org/licenses/by-nc-nd/4.0/deed.fr

Remerciements : Les auteurs remercient le $D^{r}$ Holger Flick pour sa collaboration à cet article.

Correspondance : Philipp Jud, philipp.jud@medunigraz.at

Les images cliniques sont choisies pour leur caractère particulièrement intéressant, classique ou impressionnant. Toute soumission d'image de haute résolution claire et bien identifiée doit être accompagnée d'une légende aux fins de publication. On demande aussi une brève explication (300 mots maximum) de la portée éducative des images, et des références minimales. Le consentement écrit du patient au regard de la publication doit être obtenu avant la soumission. 